\title{
Altered Maxwell equations in the length gauge
}

\author{
H. R. Reiss \\ Max Born Institute, 12489 Berlin, Germany and \\ American University, Washington, DC 20016-8058, USA
}

(7 April 2013)

\begin{abstract}
The length gauge uses a scalar potential to describe a laser field, thus treating it as a longitudinal field rather than as a transverse field. This distinction is revealed in the fact that the Maxwell equations that relate to the length gauge are not the same as those for transverse fields. In particular, a source term is necessary in the length-gauge Maxwell equations, whereas the Coulomb-gauge description of plane waves possesses the basic property of transverse fields that they propagate with no source terms at all. This difference is shown to be importantly consequential in some previously unremarked circumstances; and it explains why the Göppert-Mayer gauge transformation does not provide the security that might be expected of full gauge equivalence.
\end{abstract}




\section{INTRODUCTION}

It is shown here that the length gauge (LG) is based on a different set of Maxwell equations than those that relate to plane-wave fields. These differences can generate errors, for example, in applications of the "simpleman" method[1]. It is also shown that the squared vector potential $\mathbf{A}^{2}(t)$ for a plane wave field in the dipole approximation, cannot be transformed away despite the usual rule about quantities in the Hamiltonian that depend only on the time. These problems arise from a excessively strong form of the dipole approximation, necessary in the LG, that requires the complete absence of a magnetic field and the restriction of electric fields to time dependence alone. Working within the Coulomb gauge (CG), a much milder form of the dipole approximation can be used that makes possible some simplifications (but only when rigorously applicable) while still retaining the proper Maxwell equations. Employment of the LG for laser problems is equivalent to the treatment of plane-wave fields as if they were quasistatic electric (QSE) fields. An important distinction between plane-wave fields and QSE fields is that plane-wave fields have the unique property of propagation in the absence of sources. In contrast to this feature, QSE fields require sources, mandating the existence of a "virtual" source in the LG, that is not actually present in the laboratory. It is shown that this virtual source can introduce unphysical anomalies when using the Göppert-Mayer gauge transformation[2] to introduce the LG.

For single-active-electron atomic ionization problems, the LG interaction Hamiltonian has the form

$$
H_{I}^{L G}=\mathbf{r} \cdot \mathbf{E}(t)
$$

and the CG interaction Hamiltonian is

$$
H_{I}^{C G}=\frac{1}{c} \widehat{\mathbf{p}} \cdot \mathbf{A}(t, \mathbf{r})+\frac{1}{2 c^{2}} \mathbf{A}^{2}(t, \mathbf{r}),
$$

where $\widehat{\mathbf{p}}$ is the momentum operator, $\mathbf{E}(t)$ is the dipole-approximation electric field, and $\mathbf{A}(t, \mathbf{r})$ is a fully-stated vector potential. Atomic units are used, and electromagnetic quantities are in Gaussian units. Dipole approximation is here taken to mean that the phase $\omega t-\mathbf{k} \cdot \mathbf{r}$ of a plane wave ( $\mathbf{k}$ is the propagation vector) is approximated by $\omega t$ alone. This will be referred to as the strong form of the dipole approximation since it has the consequence that

$$
\mathbf{E}=\mathbf{E}(t), \quad \mathbf{B}=0
$$


An important feature of the strong-form dipole approximation is that the fundamental Lorentz invariant $\mathbf{E}^{2}-\mathbf{B}^{2}$ has a positive value

$$
\left(\mathbf{E}^{2}-\mathbf{B}^{2}\right)^{L G}>0,
$$

in the $L G$ rather than the zero value

$$
\left(\mathbf{E}^{2}-\mathbf{B}^{2}\right)^{P W}=0
$$

that characterizes plane-wave (PW) fields. (The descriptions "plane-wave field" and "transverse field" will be used interchangeably.) This basic departure from plane-wave behavior becomes increasingly important as the field strength increases, thus explaining why this distinction seems not to be a source of difficulty in the context of conventional perturbative Atomic, Molecular, and Optical (AMO) physics. It can, however, be critically important in very intense laser fields 3 - 5$]$.

The Göppert-Mayer 2] gauge transformation ostensibly connects the LG and the CG and makes it possible to achieve (in many problems) valid results in both gauges. The LG is useful because it requires only a scalar potential, which is generally much easier to employ than is a vector potential. However, the limitation to a scalar potential has several consequences that are often overlooked. The $\mathbf{r} \cdot \mathbf{E}(t)$ interaction Hamiltonian (11) is exactly the same as pertains to the presence of a quasistatic electric (QSE) field. The limitation to a scalar potential means that a propagating solution does not exist at all. A QSE field is a longitudinal field; an entity that is fundamentally different from the transverse field that it is intended to mimic. A QSE field oscillates with time, but it does not propagate through space with time in the manner of a plane-wave field. There is no solution of the Maxwell equations that describes a propagating field when only a scalar potential exists. The Göppert-Mayer (GM) gauge transformation essentially amounts to the use of a longitudinal field as a proxy for a transverse field.

In her 1931 paper[2], Göppert-Mayer remarks that the wavelength of the field should be much larger than the size of the atomic system with which it interacts. This is not a limitation that can be deduced from the LG itself; rather, it comes from the fact that Göppert-Mayer was aware that she was using an approximation to a propagating field, and that this approximation is not applicable if the wavelength of the propagating field is short enough to probe the details of the atomic structure. However, she makes no mention of a 
limitation at low frequencies. That omission remains largely in place at present, despite the fact that, in the modern laser environment, it is the low frequency limitation that is of far greater importance[3, 4] than the high frequency limit.

One of the most important conclusions reached below, however, is that gauge-related differences exist even within the parameter space in which the dipole approximation is nominally valid.

Section II discusses the Lorentz invariants that can be used to identify classes of electromagnetic phenomena. This is of critical importance because the LG and the CG are associated with different electromagnetic classes. These different classes are governed by different sets of Maxwell equations. In particular, the solution of the classical Maxwell equations employed in the theory of rescattering phenomena[1] does not relate to underlying properties of plane waves, but rather to the often quite different properties of quasistatic electric fields.

A vital element in the discussion of relevant Maxwell equations arises from the realization that a description of laser-induced phenomena in the LG requires the introduction of a virtual source current that is not actually present in the laboratory. In some circumstances, this virtual current can introduce unphysical energy or angular momentum into a problem.

A basic imperative that emerges in this section on electrodynamics is the necessity to retain the squared vector potential $\mathbf{A}^{2}$ even when the dipole approximation appears to make it a function only of time. The familiar rule that an additive quantity in the Hamiltonian or Lagrangian function plays no role in the equations of motion is not applicable when that term is large and arises when using an approximate treatment of plane wave fields.

The preservation of basic CG properties even when dipole-approximation forms are justified is verified by formulating problems such as the Strong-Field Approximation (SFA) in a fully relativistic context [6, 7], and then demonstrating that the dipole-approximation result follows as a limit of the fully relativistic case [8-10]. The results support the need to retain $\mathbf{A}^{2}$ when it is of significant magnitude.

Section III examines those problems where these basic differences in the LG and the CG can cause major errors to occur. For example, the simpleman method[1] can give erroneous predictions with circularly polarized fields even within the domain of field parameters where the dipole approximation is justified in the CG. Problems also arise in the significantly different ranges of applicability of the Strong Field Approximation (SFA) in the LG and in 
the CG. For example, the SFA in the dipole-approximated CG can give excellent results for plane-wave fields of quite high frequency[11-13], whereas the LG leads to tunneling models that have no applicability at all for high frequencies[3].

Section IV discusses the limitations in the LG description of plane-wave phenomena when the putative Volkov solution (an explicitly propagating-wave solution) is employed in a gauge that does not possess a traveling-wave property.

Finally, Section V summarizes the essential findings of the paper.

\section{BASIC ELECTRODYNAMIC BACKGROUND}

The characteristics of an electromagnetic field inferred from the Lorentz invariants associated with different field configurations are reviewed, since they serve as a prelude to examining the Maxwell equations directly. Gaussian units are used to achieve clarity through simplicity of notation.

\section{A. Lorentz invariants}

The basic Lorentz invariants that characterize an electromagnetic field are inferred from the field tensor, which is

$$
F^{\mu \nu}=\partial^{\mu} A^{\nu}-\partial^{\nu} A^{\mu}
$$

where $A^{\mu}$ is the 4 -vector potential that describes the electromagnetic field. The relativistic conventions employed are those of Jackson[14]. A dual tensor $\mathcal{F}^{\mu \nu}$ can also be defined by acting on the field tensor with the completely antisymmetric 4-space tensor $\epsilon^{\mu \nu \rho \lambda}$. The two Lorentz invariants that can be formed from scalar products of these tensors are [14]

$$
\begin{aligned}
& F^{\mu \nu} F_{\mu \nu}=-2\left(\mathbf{E}^{2}-\mathbf{B}^{2}\right), \\
& \mathcal{F}^{\mu \nu} F_{\mu \nu}=-4 \mathbf{E} \cdot \mathbf{B},
\end{aligned}
$$


often referred to simply as the $\mathbf{E}^{2}-\mathbf{B}^{2}$ and $\mathbf{E} \cdot \mathbf{B}$ scalar Lorentz invariants. They make possible the simple chart

\begin{tabular}{|c||c|c|}
\hline & $\mathbf{E}^{2}-\mathbf{B}^{2}$ & $\mathbf{E} \cdot \mathbf{B}$ \\
\hline \hline constant electric field & $\mathbf{E}^{2}$ & 0 \\
\hline plane wave field & 0 & 0 \\
\hline constant magnetic field & $-\mathbf{B}^{2}$ & 0 \\
\hline
\end{tabular}

The $\mathbf{E} \cdot \mathbf{B}$ invariant is zero for all three cases, albeit for different reasons. It is the $\mathbf{E}^{2}-\mathbf{B}^{2}$ invariant that makes the important distinctions: it is positive-definite for the constant electric field (and the QSE field), negative definite for the constant magnetic field, and zero for the plane wave field. The stronger the field, the more important the distinctions become. The plane wave field is a transverse field that has the properties

$$
|\mathbf{E}|=|\mathbf{B}|, \quad \mathbf{E} \perp \mathbf{B}
$$

that underlie the entries in (9). It is called transverse because the electric and magnetic fields are both perpendicular to the direction of propagation of the field as well as to each other. The constant electric field is the limiting case of a QSE field or longitudinal field, where the sole preferred direction in the physical problem is the direction of the electric field. From the chart (9) it can be said that the constant electric field is as different from a plane wave as is the constant magnetic field.

It is emphasized that the basic properties of a plane wave field as expressed in (9) and (10) cannot be replicated by a scalar potential.

\section{B. Maxwell equations}

The Maxwell equations for the vacuum are

$$
\begin{aligned}
\nabla \cdot \mathbf{E} & =4 \pi \rho, \\
\nabla \cdot \mathbf{B} & =0 \\
\nabla \times \mathbf{B}-\frac{1}{c} \frac{\partial}{\partial t} \mathbf{E} & =\frac{4 \pi}{c} \mathbf{J}, \\
\nabla \times \mathbf{E}+\frac{1}{c} \frac{\partial}{\partial t} \mathbf{B} & =0
\end{aligned}
$$

where $\rho$ and $\mathbf{J}$ are source terms, being respectively a charge density and a current density. 


\section{Maxwell equations for plane waves}

Plane waves have the distinction that, once formed, they can propagate without limit in the complete absence of sources. The Maxwell equations appropriate to plane waves are found from Eqs. (11) - (14) by giving zero values to the sources. This gives the Maxwell equations for plane waves as

$$
\begin{aligned}
\nabla \cdot \mathbf{E} & =0, \\
\nabla \cdot \mathbf{B} & =0, \\
\nabla \times \mathbf{B}-\frac{1}{c} \frac{\partial}{\partial t} \mathbf{E} & =0, \\
\nabla \times \mathbf{E}+\frac{1}{c} \frac{\partial}{\partial t} \mathbf{B} & =0 .
\end{aligned}
$$

\section{Dipole approximation}

When plane waves interact with a bound system whose size is much smaller than a wavelength $\lambda$ of the field, this sets an upper limit on the frequency for which the dipole approximation is applicable, given by

$$
\lambda \gg a_{0}, \text { or } \omega \ll \frac{2 \pi c}{a_{0}},
$$

where $a_{0}$, the Bohr radius, characterizes the size of an atom. In atomic units (a.u.), used henceforth, this is just

$$
\omega \ll 2 \pi c .
$$

A lower limit on the frequencies for which the dipole approximation can be applied comes from the onset of specific effects of the magnetic field, given by [3, 7]

$$
\omega \gg \frac{1}{2}\left(\frac{I}{c}\right)^{1 / 3}
$$

for linear polarization, where $I$ is the field intensity. This lower frequency limit on the dipole approximation does not occur naturally in the LG, where it is often overlooked.

Göppert-Mayer, in her famous 1931 paper[2], specified only the upper limit on the frequency that is given by Eq. (19). This overlooking of the need to fully consider the requirements for complete neglect of the magnetic field is not surprising for a 1931 paper. However, that 80-year-old oversight continues to prevail in almost all current work. 
The dipole approximation is often taken to be characterized by the application of the strong form as given in Eq. (3). These conditions are not possible in terms of Eqs. (15)

- (18). This means that the dipole approximation for a plane-wave field cannot be stated simply as Eq. (3).

It must be kept in mind that plane waves are unique in that they are the only propagatingwave solution of the Maxwell equations. Transverse fields cannot propagate unless the magnetic field is present and equal in magnitude to the electric field. The conditions (3) are an over-simplification when applied to plane waves, whereas the properties (10) are always valid and necessary for plane waves. When a specific quantum matrix element is to be evaluated, it may be possible to employ the approximations (3) without affecting the value of the matrix element. It is also true that, even when $|\mathbf{B}|=|\mathbf{E}|$, the classical force exerted by the magnetic field on a charged particle can be negligible compared to the electric force because of the Lorentz force

$$
\mathbf{F}=q\left(\mathbf{E}+\frac{\mathbf{v}}{c} \times \mathbf{B}\right)
$$

exerted on a particle of charge $q$. Nevertheless, plane waves are always associated with zero values for the $\mathbf{E}^{2}-\mathbf{B}^{2}$ and $\mathbf{E} \cdot \mathbf{B}$ invariants listed in the chart (9). The upper limit on the frequency for which the conditions (3) are harmless approximations for plane wave phenomena is given by Eq. (19), and the lower limit comes from Eq. (20). Within those bounds, the actual presence of a magnetic field with $|\mathbf{B}|=|\mathbf{E}|$ does not have major consequences for linear polarization problems beyond that of maintaining the propagation property of a plane wave. The circular polarization case (and thus elliptical polarization approaching circular) involves more restrictive requirements to be examined below.

\section{E. Maxwell equations for the length gauge}

The length gauge explicitly obeys the conditions in Eq. (3)). To state the appropriate Maxwell equations, it is necessary to return to the complete expression of the Maxwell equations in Eqs. (11) - (14). The conditions (3) reduce the four Maxwell equations (11) (14) to the single equation

$$
\frac{\partial}{\partial t} \mathbf{E}(t)=-4 \pi J_{v} .
$$

The subscript $v$ has been appended to the current term to indicate that it is a virtual current that does not really exist in the laboratory; it is necessary in order to support a non-trivial 
result for the behavior of a charged particle described in the length gauge.

An essential property of the LG is that it requires only a scalar potential, as shown in Eq. (11). It is this simplicity that makes it so attractive to use. An immediate inference is that the magnetic field is absent. The single Maxwell equation required for the LG is Eq. (21). Even when $\mathbf{E}$ has some time dependence, so that $\mathbf{E}=\mathbf{E}(t)$, it remains possible to neglect the magnetic field over a significant range of laser field frequencies and intensities. This range is illustrated in Fig. 1, taken from Ref. [3]. The upper frequency limit in Fig. 1 actually refers to tunneling theories rather than to the less restrictive Eq. (19), but most analytical strong-field approximations that employ the LG also employ tunneling concepts. This will not be the subject of further comment since it is the lower frequency limit on the applicability of the dipole approximation that is often neglected and is of greater interest in laser applications. The lower frequency limit (20) on the applicability of the dipole approximation is determined by the onset of magnetic field effects on a free electron [3, 7].

Following a criterion set some years ago[15, 16], it has become a widely accepted practice to judge the validity and reliability of analytical approximations in plane-wave phenomena by comparing their behavior at zero frequency with known constant electric field results. This criterion is not applicable to plane waves. Plane waves do not have a zero frequency limit because that would entail a divergent ponderomotive energy. This is discussed below.

The shaded area in Fig. 1 is where dipole-approximation results can be employed to describe plane wave phenomena (at least for linear polarization). This can be dubbed the "oasis", where dipole-approximation theories for the description of laser-induced phenomena can be accurate. A fundamental difficulty is that, starting at any point within the oasis, there is no path by which zero frequency can be approached without crossing the lower-frequency limit of the oasis, and entering into a domain where LG approaches to laser phenomena are not applicable. This inference is clear, since zero frequency implies the first entry in the chart (91), which is not descriptive of plane wave fields at all. Another way to understand this is to consider the behavior of the ponderomotive potential $U_{p}$, which is a fundamental property of plane wave fields $[17-20]$. The ponderomotive potential depends on frequency as

$$
U_{p} \sim \omega^{-2}
$$

which means that it is not possible to pass to a zero frequency for a plane wave.

It is often explicitly stated that the zero frequency limit of a plane wave field exists, and 


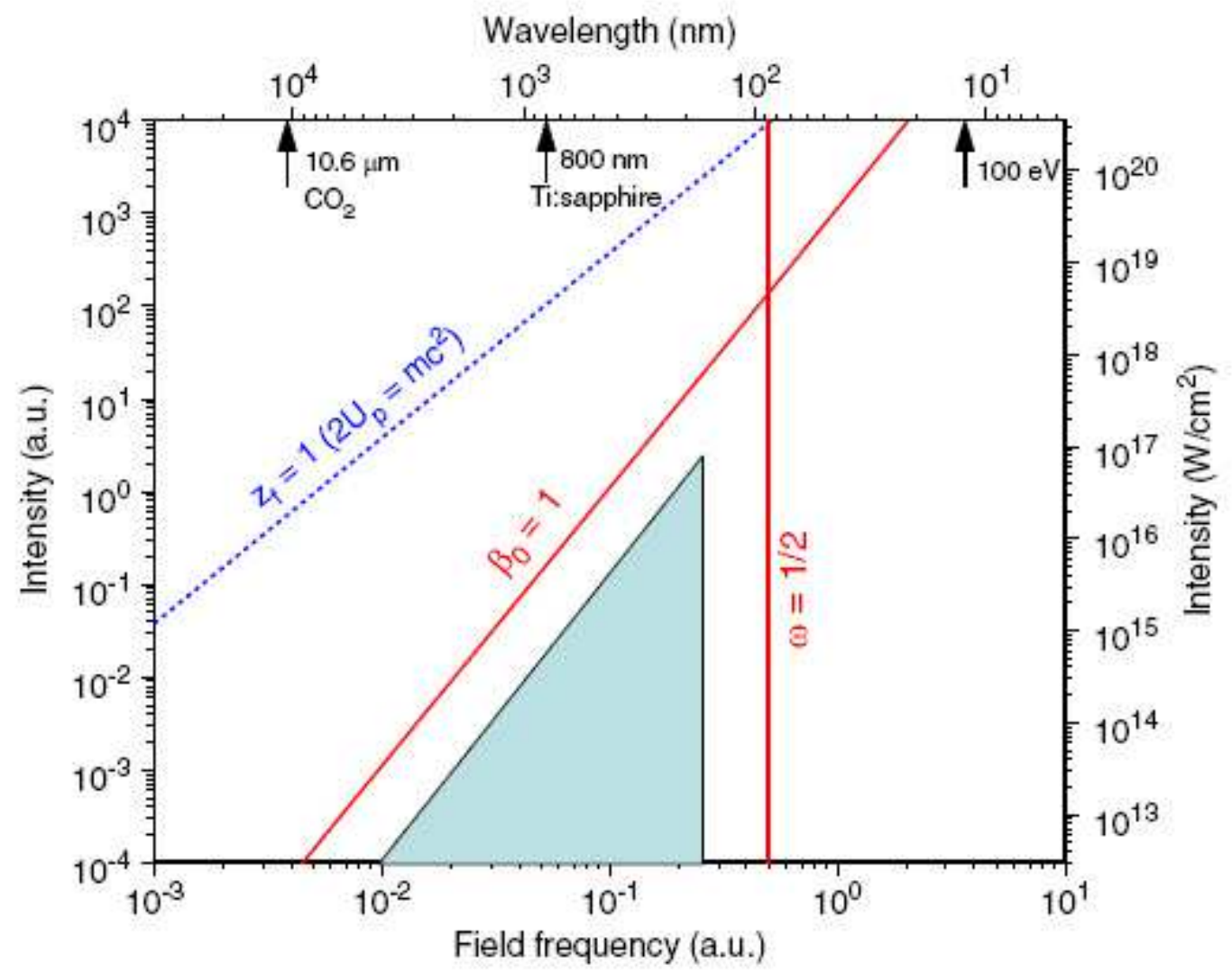

FIG. 1: This figure, taken from Ref. [3], shows the common domains of applicability for the LG and the CG as the shaded area, referred to in the text as the "oasis". The line labeled $\beta_{0}=1$ corresponds to the onset of magnetic field effects at lower frequencies, as given by Eq. (20). The line labeled $\omega=1 / 2$ denotes an upper frequency limit set by the applicability of tunneling methods. A less restrictive condition for the upper frequency limit is given by Eq. (19).

that it is a static electric field. That statement is motivated by LG concepts. It is not correct. As one of many examples to be found in the strong-field literature, a recent paper states[21]: "In adiabatic tunneling the laser field is treated as if it were a static field ... It is rigorously valid for long wavelengths ...". This quote starts by citing correctly the essential property of the LG as treating the laser field as if it were a static electric field or a QSE field. However, the remark that it is "rigorously valid" for long wavelengths misidentifies the limit of a QSE field as $\omega \rightarrow 0$ as being a legitimate limit of a plane wave field. The same source continues with the statement: "Nonadiabatic tunneling refers to deviations that arise at higher frequencies ...", thus retaining the inadequate 1931 view of Göppert-Mayer[2] that 
the dipole approximation possesses only a high frequency limit.

\section{F. Ponderomotive potential}

A fundamental property of a charged particle in a laser field is that it exhibits a ponderomotive potential $U_{p}$ due to the presence of the field. See Sections 6.2.2 and 7 of [19], and Section IV of [20] for the critical role that $U_{p}$ plays in distinguishing transverse fields from other types of electromagnetic phenomena. The ponderomotive potential depends on $A^{\mu} A_{\mu}$ in general, or on $\mathbf{A}^{2}$ in the $\mathrm{CG}$. In most contemporary laser experiments the dipole approximation applies, which makes $\mathbf{A}^{2}$ appear to depend only on the time: $\mathbf{A}^{2}=\mathbf{A}^{2}(t)$. A basic theorem in mechanics asserts that any term in the Hamiltonian or Lagrangian function that is a function only of the time can be removed without any effect on the equations of motion. However, $U_{p}$ can be very large in practical examples where the dipole approximation seems to be valid by most criteria, but where the elimination of $\mathbf{A}^{2}(t)$ would make a huge difference were it not retained. For example, at a wavelength of $800 \mathrm{~nm}$ and an intensity of $10^{15} \mathrm{~W} / \mathrm{cm}^{2}, U_{p}$ amounts to about $60 \mathrm{eV}$. The ponderomotive potential is an essential aspect of plane-wave phenomena, and eliminating it would make a drastic and unphysical alteration in the description of the effects of laser fields. The question of the neglect or retention of $\mathbf{A}^{2}(t)$ has been debated for many years. It led to the recommendation[6] that all strong field problems should be formulated in relativistic terms on the grounds that strong laser fields make the field a dominant aspect of a problem. Photon fields propagate with the velocity of light, so that they impose a relativistic context upon the entire problem. Starting with a relativistic formalism and then passing to a dipole approximation when it is warranted should resolve such difficulties as the retention of $\mathbf{A}^{2}(t)$. That suggestion[6] had little effect on the strong-field community, and the current proposal to rely on the need to sustain plane-wave properties such as the relativistic invariants like $F^{\mu \nu} F_{\mu \nu}$ and the onset of explicit magnetic field effects is a more robust approach.

\section{THE "SIMPLEMAN" THEORY}

The simpleman theory is so called because it envisions elementary classical behavior of a charged particle in an electric field as the underlying explanation for many laser-caused 
phenomena. A clear statement of this approach is contained in Ref. [1]. At the heart of the idea is the set of classical solutions for an electron in a sinusoidally varying electric field. These solutions correspond to the Maxwell equation (21). That is, they are the result of the virtual current $J_{v}$.

\section{A. Linear polarization}

The case of linear polarization presents the opportunity to compare the predictions of the LG and the CG. An important application of the simpleman method is for the explanation of high harmonic generation (HHG). For linear polarization of the laser, the scenario envisioned in the LG is that atomic ionization occurs by a tunneling process and, after exiting the tunnel, the photoelectron has its subsequent motion governed by the classical solutions of (21). The motion is oscillatory and primarily along the direction of the electric field, so that upon reversal of the field direction the electron is driven back to the parent atom. Recombination can then occur, with the emission of a photon representing the initial binding energy plus an additional energy corresponding to a multiple of the frequency $\omega$ of the driving field.

An explanation in terms of the $\mathrm{CG}$ would be similar. The primary difference is that, in the LG, the electron is "driven" by the applied field, whereas, in the CG the laser field is viewed properly as a transverse field that cannot transfer net energy to a free electron, even though there is a regular sharing of energy between the field and the charged particle during any cycle. An equivalent way to state this is to remark that the LG treats the laser field as if it were a quasistatic electric field rather than as a proper plane-wave field. The difference is most easily understood as a contrast between the "constant electric field" and the "plane wave field" entries in the chart (9). In a pure plane-wave situation (that is, with no residual Coulomb effects), the trajectory followed by an electron would be determined entirely by the initial conditions of its entry into the field. Such trajectories are well known. The most complete source for this information is probably the article by Sarachik and Schappert[22]. The results given there are fully relativistic because a plane wave field is a relativistic object. However, if the nonrelativistic limit is taken of the trajectory information given in Ref. [22], then the electron motion is a simple oscillation with exactly the same amplitude in the electric field direction as is found for the LG case. The relativistic plane wave solution has 
the well-known "figure- 8 " configuration in the frame of reference in which the electron is at rest on the average over a full cycle, with the long axis in the direction of the electric field and the short axis in the propagation direction of the field. In the nonrelativistic limit, the amplitude in the propagation direction approaches zero, and the LG and CG predictions for the classical orbit of the free electron become identical.

\section{B. Circular polarization in the LG}

For circular polarization, the LG and the CG give very different results. The LG case is considered first. Equations (2) and (3) of Ref. [1] provide the necessary information for the classical trajectory of an electron that exits an atom by tunneling ionization. This sets initial conditions at zero velocity at the exit of the tunnel, which can be taken to be at the origin of coordinates. The trajectory is then found to be

$$
\begin{aligned}
x & =x_{0}(1-\cos \omega t), \\
y & =x_{0}(\omega t-\sin \omega t), \\
x_{0} & =\mathcal{E}_{0} / \omega^{2},
\end{aligned}
$$

for a circularly polarized field about the $z$ direction as the axis. The quantity $\mathcal{E}_{0}$ is the amplitude of the electric field of frequency $\omega$. The trajectory is plotted in Fig. 2 for motion in an electric field corresponding to a wavelength of $800 \mathrm{~nm}$ at $10^{14} \mathrm{~W} / \mathrm{cm}^{2}$. It shows an oscillation of the electron of constant amplitude in one direction in the plane of motion, but with a trajectory in the other direction in the plane of motion that has been described as "walking away" from the ion. The most interesting aspect of this motion comes from an examination of the angular momentum of the electron as measured from the position of the residual ion. The angular momentum is simply

$$
\begin{aligned}
l & =x v_{y}-y v_{x} \\
& =\omega x_{0}^{2}[\omega t \sin \omega t-2 \cos \omega t(1-\cos \omega t)],
\end{aligned}
$$

as follows from Eqs. (23) and (24). This is shown in Fig. 3. After a few cycles, the angular momentum is dominated by the first term in Eq. (26), giving

$$
l \approx \omega^{2} x_{0}^{2} t \sin \omega t
$$




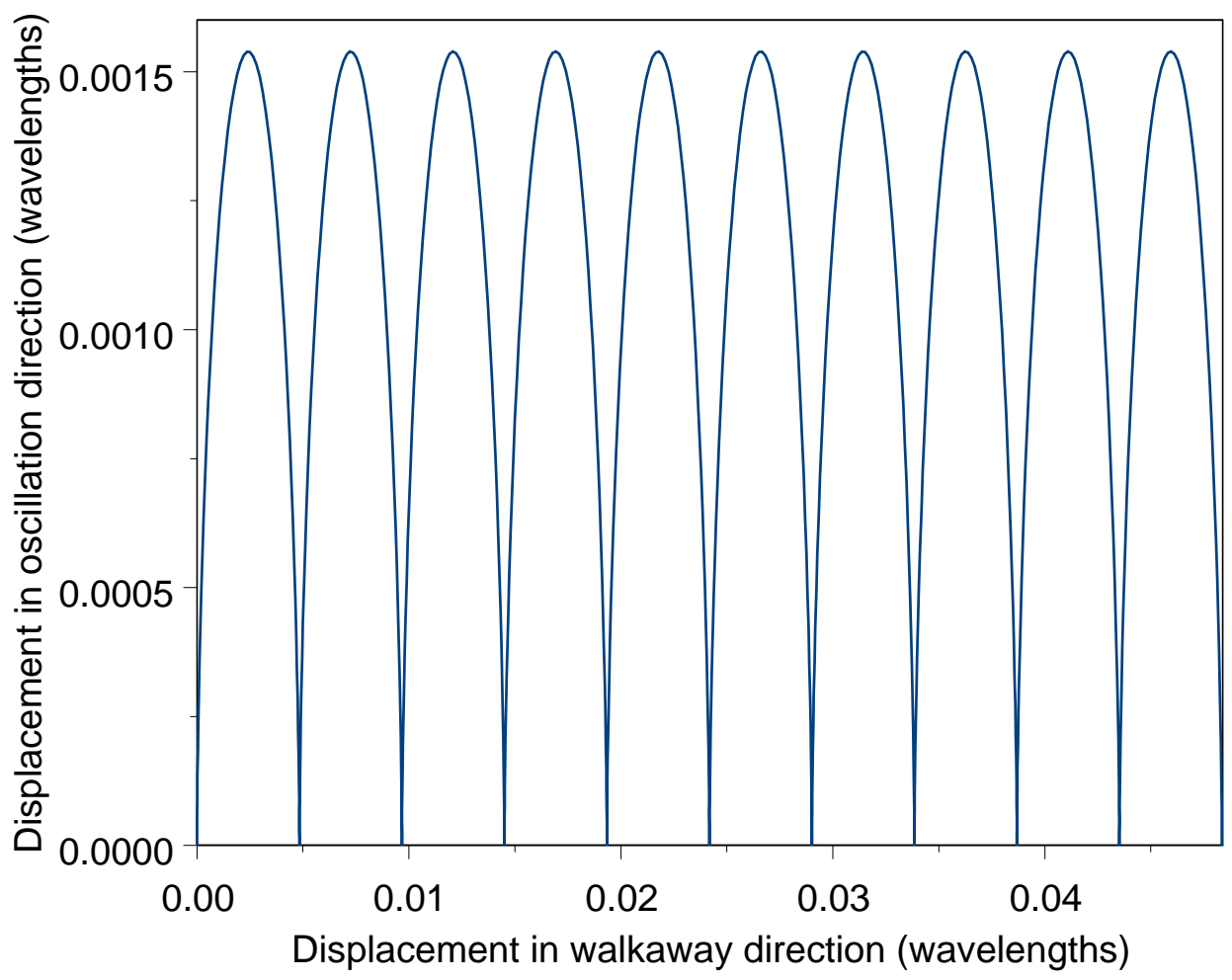

FIG. 2: This figure shows the LG particle trajectory as a photoelectron "walks away" from the atom after ionization by a circularly polarized field. Ten constant-intensity cycles are shown. The "walkaway" phenomenon is found only in the LG, and is a result of the tunneling view of ionization that is appropriate in the LG, but not in the CG.

a form that makes explicit how the amplitude of the angular momentum oscillation increases linearly with time. Figures 2 and 3 plot the motion for ten cycles at constant amplitude.

The curious feature in Fig. 3 is the continuous buildup of angular momentum, following the behavior shown in Eq. (27). In a real ionization event generated by a circularly polarized laser field (when there is no difference between the angular momentum states of the initial atom and the remnant ion), the post-ionization angular momentum should simply be the angular momentum contained in the number of photons required to ionize. For the stated parameters, the ponderomotive energy $U_{p}$ of the electron in the field is about 0.22 a.u. If the atom being ionized is ground-state hydrogen, the minimum number of photons required 


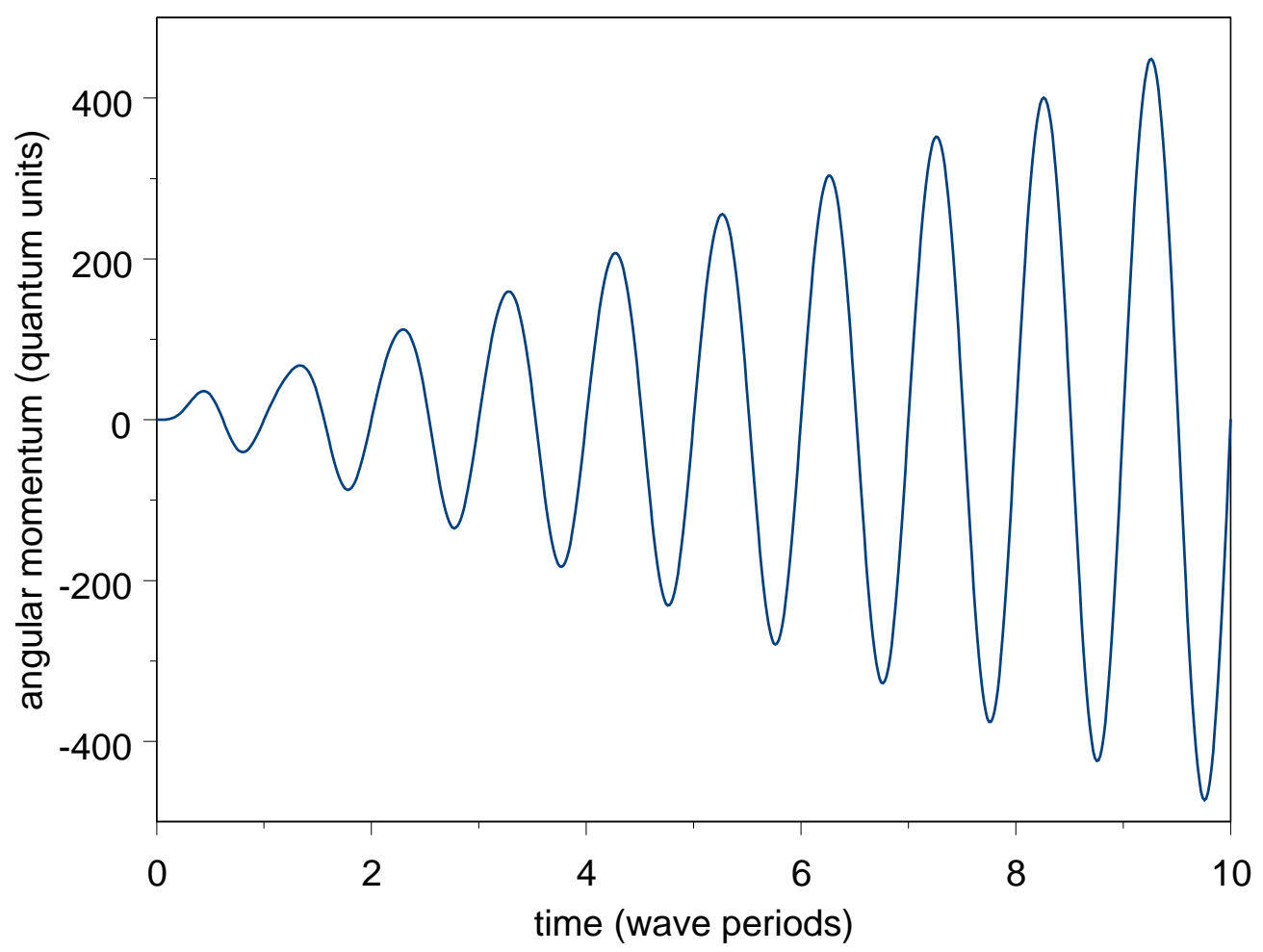

FIG. 3: The angular momentum associated with the "walkaway" phenomenon of Fig. 2 is shown here. The angular momentum is calculated with respect to the atom location as the center. This oscillatory behavior is physically impossible, since a circularly polarized laser can impart only a single sense of rotation.

for ionization is

$$
n_{0}=\left\lceil\frac{E_{B}+U_{p}}{\omega}\right\rceil=\left\lceil\frac{.5+.22}{.057}\right\rceil=13,
$$

and the most probable kinetic energy of a photoelectron after ionization by a circularly polarized laser field is a further $U_{p}$. The most probable number of photons absorbed for ionization is thus

$$
n=\left\lceil\frac{E_{B}+2 U_{p}}{\omega}\right\rceil=\left\lceil\frac{.5+.44}{.057}\right\rceil=17 .
$$

The bracket symbol that appears in Eqs. (28) and (29) is the ceiling function, meaning the smallest integer containing the quantity within the bracket. Each circularly polarized photon possesses one quantum unit of angular momentum, and all of these angular momenta are 
aligned, meaning that the photoelectron should possess about 17 quantum units of angular momentum.

Figure 3 shows a continuous increase of angular momentum, reaching more than $400 \hbar$ after ten cycles. Not only is this a completely unrealistic magnitude, but the figure shows that the angular momentum oscillates between positive and negative values. This is not a physically possible outcome, since a circularly polarized field has a unique sense of rotation and can impart only one sign of angular momentum to the photoelectron.

\section{Circular polarization in the $\mathrm{CG}$}

The relevant Maxwell equations in the CG are Eqs. (15) - (18). There are no external sources. In particular, there is no virtual current density $J_{v}$. A photoelectron will continue the trajectory which is imparted as the end result of ionization. This must therefore be a circular orbit centered on the remnant ion and possessing the energy and angular momentum of a classical orbit. The means by which this can happen are strongly suggested by a Umatrix calculation of the ionization of ground-state hydrogen by a single cycle of a circularly polarized laser field[23]. The U matrix tracks the progress of a particle with time, and approaches the S matrix for large times. The result of this single-cycle calculation is that the probability density for the electron moves out to the classical orbit position as the field amplitude increases. As the amplitude of the field subsequently decreases, some of the electron probability distribution returns to the distribution of the neutral atom, but a portion of it is missing. This is the portion that has been ionized.

A similar scenario, but in the long-pulse regime, is suggested by the calculation of a correction to the SFA for ionization by circularly polarized light [24]. The SFA regards the final state of the electron as being that of a Volkov electron; that is, an electron moving under the influence of the plane-wave field. The field is regarded as the dominant influence on the free electron, with a neglect of residual effects of the Coulomb field of the atom. The correction turns out to be the influence of the Coulomb field on an electron circulating around the ion in a circular path of classical radius in the background field.

The recent matching[25] of a fully relativistic theory to experiment[26] confirms the picture of a photoelectron created by circularly polarized light entering into a circular orbit around the ion. 
The underlying reason for both the long-pulse and short-pulse scenarios comes from the fact that a free electron cannot exchange net energy with a plane-wave field. During any single cycle, there is an exchange of energy between the electron and the field but, if the field intensity is maintained constant, subsequent cycles replicate the cycle history exactly, with no gain in overall energy. The final state of the photoelectron must thus represent a photoelectron that satisfies all necessary conservation conditions, including the conservation of angular momentum that is so glaringly violated in the LG approach to ionization by a circularly polarized field. The LG acquires from the virtual current density $J_{v}$ the energy and angular momentum required to provide the contrasts in Fig. 3,

Figures 4 and 5 relate to the first two of the ten cycles shown in Figs. 2 and 3 , Figure 4 compares the LG with the CG prediction for the motion of the photoelectron. Were the two axes scaled the same, then the CG motion would be circular. Figure 5 shows the angular momentum in the CG and LG cases for the same two cycles. The CG - LG contrasts in Figs. 4 and 5 are extreme.

\section{Observed qualitative difference between LG and CG for circular polarization}

The "walkaway" phenomenon discussed above follows from the general tunneling behavior that the direction of the electric field is dominant in the ionization process. With circular polarization, the electric field is always in the radial direction. In the CG, the long-pulse case simply leads to cylindrical symmetry about the propagation direction, but the single-cycle U-matrix calculation [23] is more explicit. There it is found that it is the vector potential A that is the dominant influence, and the vector potential is $\pi / 2$ out of phase with the electric field. That is, when the electric field is in the radial direction, the vector potential is in the azimuthal direction. Thus the CG is consistent [27] with the observed "donut" shape of the photoelectron angular distribution arising from ionization by circularly polarized light. See Fig. 1 of Ref. [28], as discussed in Ref. [27].

These same properties of photoelectrons arising from circularly polarized laser light are also confirmed [25] in the recent experiments by Smeenk et al.[26]. That is, when ionized by circularly polarized light, the photoelectron is emitted in a direction perpendicular to the direction of the electric field, not aligned with the electric field as implied by the LG. 


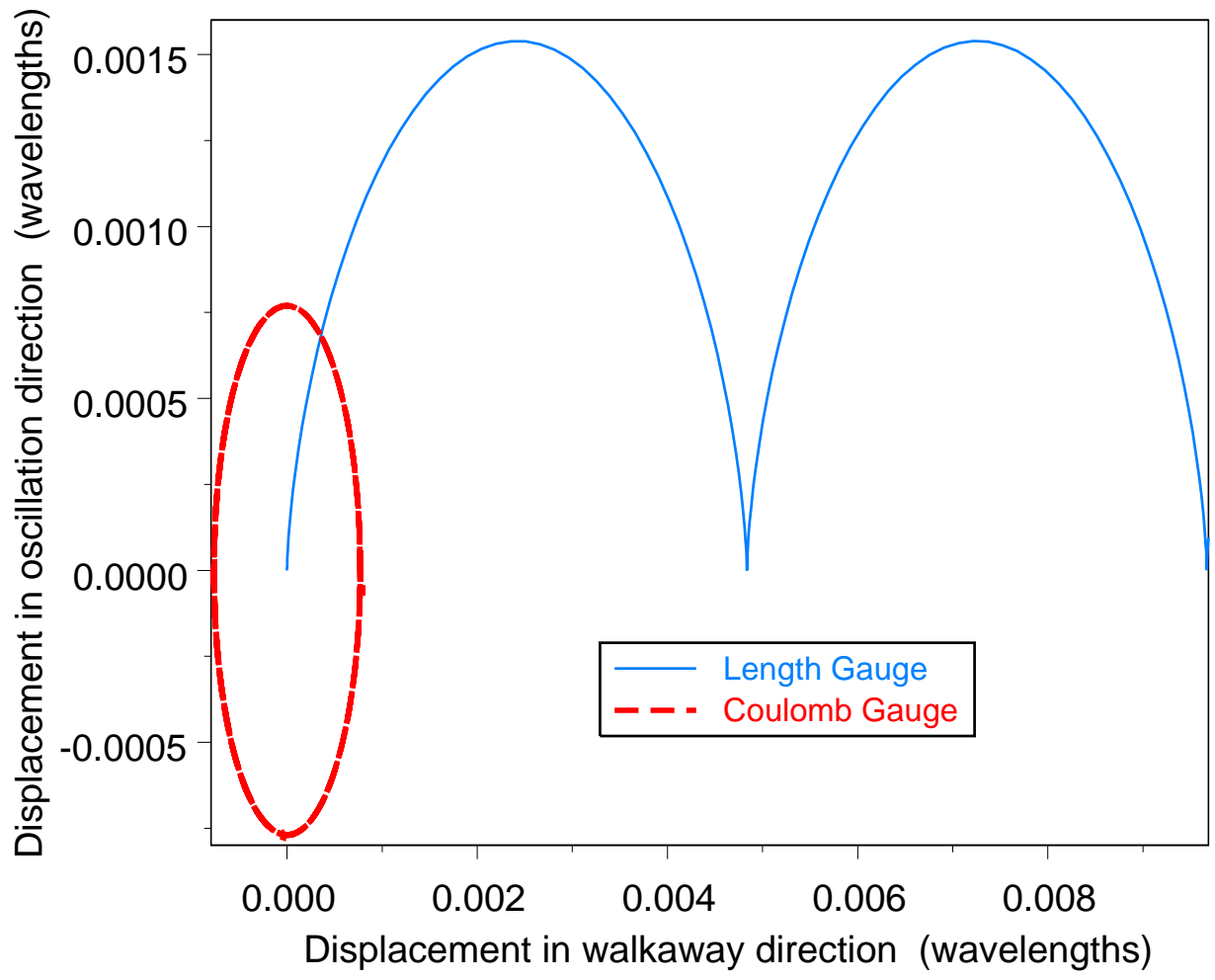

FIG. 4: The LG trajectory of the photoelectron shown here corresponds to the first two of the ten cycles illustrated in Fig. 2, The CG trajectory is a closed orbit around the remnant ion. The scales along the two axes are different. Were they to the same scale, the CG trajectory would be a circular orbit around the ion with energy and angular momentum corresponding to classical parameters for the circular polarization in this example.

\section{IV. "VECTOR POTENTIAL" IN THE LENGTH GAUGE}

The 1980 theory[29] of the present author is the only SFA theory that is formulated in the CG, with subsequent reduction to a dipole-approximation theory[6, 7]. Other putative versions of the SFA (including the Keldysh theory [30], despite the injunction against naming any tunneling theory the "SFA" as explained in Ref. [6]) employ Volkov solutions in the LG. This is a curious situation. There is no possible propagating-wave solution of the Maxwell equations that can be expressed by a scalar potential alone, yet that is the defining feature of the LG. The ostensible LG Volkov solution is formulated in the velocity gauge and then 


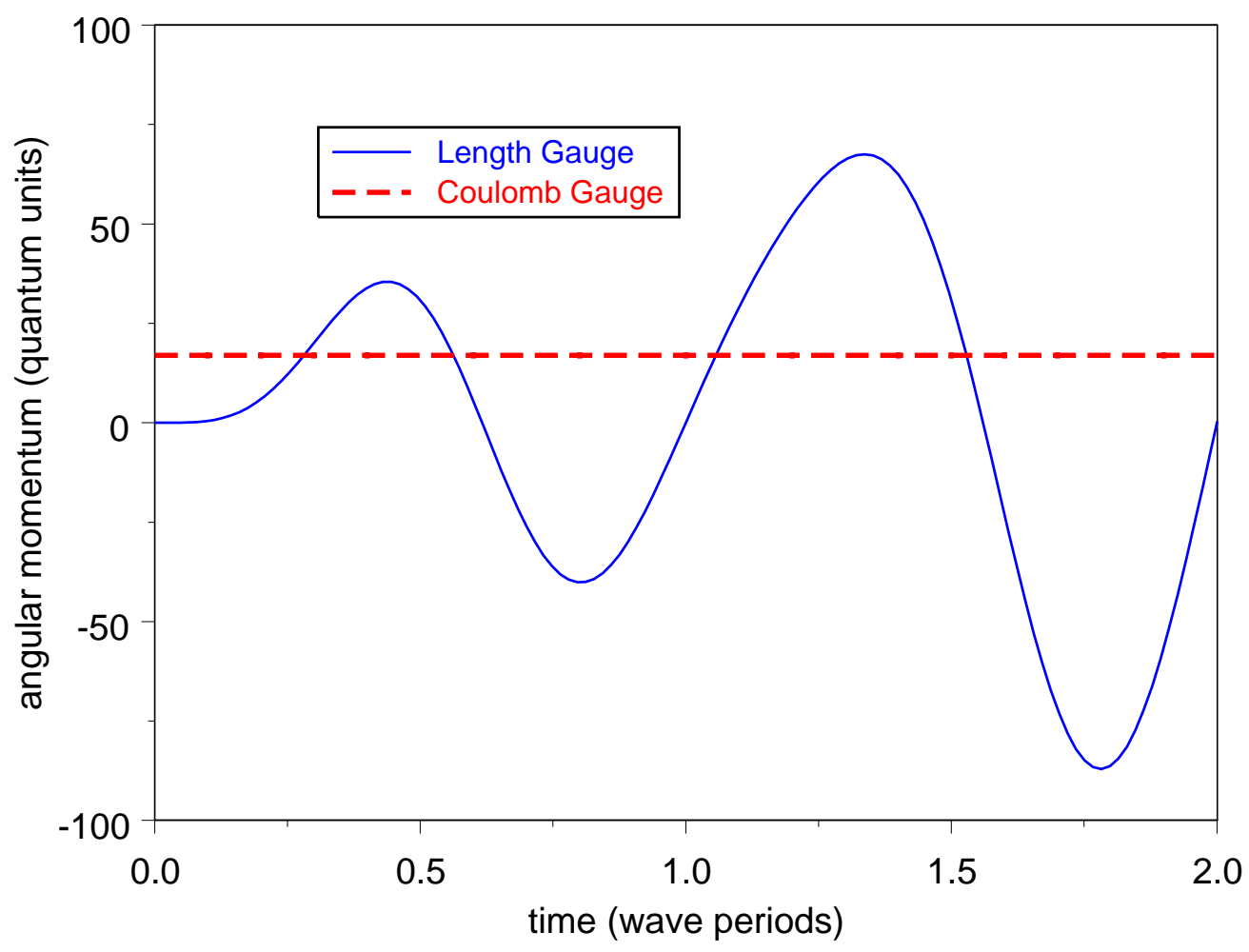

FIG. 5: As in Fig. 4, only the LG angular momentum history for the first two cycles of Fig 3 are shown here. The CG angular momentum is constant, so it appears here as a simple straight line at the value given in Eq. (29).

gauge-transformed to the LG. (The velocity gauge can be derived from the CG by employing dipole-approximation assumptions that do not alter the Maxwell equations[6, 7]. Not all velocity-gauge formulations obey that stricture.) The difficulty is that the Volkov solution itself (as well as the generating function of the gauge transformation) is expressed in terms of a vector potential. There is no vector potential in the LG. For formal purposes, it is envisioned that the dipole-approximation vector potential is really defined in terms of the electric field through the relation

$$
\mathbf{A}(t)=-\frac{1}{c} \int_{-\infty}^{t} \mathbf{E}\left(t^{\prime}\right) d t^{\prime}
$$

This makes the theory nonlocal. The vector potential at any time $t$ depends on the values of the electric field at all earlier times $t^{\prime}$. This nonlocality is the price that must be paid to 
employ the Volkov solution in a gauge that has no Volkov solution.

The approximation involved in neglecting the final-state Coulomb influence in one gauge is only indirectly related to making a similar assumption in the other gauge. The CG and the LG are based on different Maxwell equations, and the Volkov solution as expressed by a welldefined vector potential in one gauge is replaced by a nonlocal expression in the other gauge. Therefore, it is inappropriate to regard the SFA as a theory that lacks gauge invariance; a statement that occurs frequently in the strong-field literature. The SFA in the CG and the so-called SFA in the LG are two distinct approximations, and are not gauge-equivalent versions of the same approximation.

\section{SUMMARY}

Plane waves are transverse fields for which the Lorentz invariant $\mathbf{E}^{2}-\mathbf{B}^{2}=0$ is a necessity. Any theory for which $\mathbf{E}=\mathbf{E}(t)$ and $\mathbf{B}=0$ are a priori conditions is a theory that can describe longitudinal fields, but not transverse fields. Within the CG, it is possible to express a dipole-approximation result, but only when the spatial dependence of the planewave phase is demonstrably unimportant. The LG as applied to plane-wave problems is not a dipole approximation at all; rather, it amounts to the assumption that a transverse field can be approximated by a longitudinal field. The GM equivalence is not complete because the price paid for having a plane-wave field approximated by a quasistatic electric field is that the relevant Maxwell equations are quite different in the LG than they are in the CG. In particular, the LG must possess a virtual charge current density $J_{v}$ that exists only for the

purpose of permitting a nominal gauge equivalence to exist between a longitudinal field and an approximated transverse field. This virtual charge current $J_{v}$ does not have discernible effects under many circumstances, but when an ionization is caused by a circularly polarized field, the tunneling mechanism (possible in the LG but not in the CG), requires a set of initial conditions for a photoelectron that demands major inputs from $J_{v}$. These effects are completely different from anything in the CG case, and are clearly unphysical for plane waves.

The "pathology" involved in the GM gauge transformation manifests itself in several ways, some of which have just been recounted. Another manifestation is in the generating function of the gauge transformation. This function is proportional to $\mathbf{r} \cdot \mathbf{A}$, where the 
vector potential A exists only in the CG. Thus the gauge transformation, by introducing an alien element into the LG, requires some nonlocal device such as exhibited in Eq. (30). This is just another of the several symptoms of the pathology.

These fundamental differences between the LG and the CG have implications for the SFA. They mean that the approximation employed in the SFA has different consequences in the two gauges. The statement that can be found in the literature that the SFA is somehow defective because it is gauge dependent, is a non sequitur.

A simple way to summarize what has been said here is the following: The LG provides a description of quasistatic electric field phenomena, because that is all that can be described by a scalar potential alone. That is, the LG represents the first line in the chart (9). Plane wave phenomena require a vector potential, since that is necessary for the description of a transverse field or, equivalently, the description of a propagating electromagnetic field. The second entry in the chart (9) relates to this phenomenon. These are the basic differences that underlie the various problems discussed above. What has confused the situation is the assumption that Eq. (3) is an unqualified condition for both the LG and the dipole-approximated CG. This underlies the original premise of the Göppert-Mayer gauge transformation. Göppert-Mayer cannot be faulted for not seeing this problem in 1931. Perturbative AMO physics has thrived for many decades on the LG. However, Eq. (3) is not universally compatible with plane waves, and this is an important distinction in strong-field, nonperturbative physics.

[1] P. B. Corkum, Phys. Rev. Lett.71, 1994-1997 (1993).

[2] M. Göppert-Mayer, Ann. Phys. (Leipzig) 9, 273-294 (1931).

[3] H. R. Reiss, Phys. Rev. Lett. 101, 043002 (2008); 101, 159901(E) (2008).

[4] H. R. Reiss, Phys. Rev. Lett. 102, 143003 (2009).

[5] H. R. Reiss, Phys. Rev. A 75, 031404(R) (2007).

[6] H. R. Reiss, Phys. Rev. A 42, 1476-1486 (1990).

[7] H. R. Reiss, Prog. Quantum Electron. 16, 1-71 (1992).

[8] H. R. Reiss, J. Opt. Soc. Am. B 7, 574-586 (1990).

[9] D. P. Crawford and H. R. Reiss, Phys. Rev. A 50, 1844-1850 (1994). 
[10] D. P. Crawford and H. R. Reiss, Opt. Express 2, 289-294 (1998).

[11] D. I. Bondar, M. Spanner, W.-K. Liu, and G. L. Yudin, Phys. Rev. A 79, 063404 (2009).

[12] H. R. Reiss, J. Opt. Soc. Am. B 13, 355-362 (1996).

[13] H. R. Reiss, Opt. Express 8, 99-105 (2001).

[14] J. D. Jackson, Classical Electrodynamics (Wiley, New York, 1975), second edition.

[15] V. S. Popov, JETP Lett. 70, 502-507 (1999).

[16] G. F. Gribakin and M. Yu. Kuchiev, Phys. Rev. A 55, 3760-3771 (1997).

[17] T. W. B. Kibble, Phys. Rev. 150, 1060-1069 (1966).

[18] P. H. Bucksbaum, M. Bashkansky, and T. J. McIlrath, Phys. Rev. Lett. 58, 349-352 (1987).

[19] H. R. Reiss, J. Mod. Optics 59, 1371-1383 (2012).

[20] J. Schwinger, Phys. Rev. 82, 664-679 (1951).

[21] L. Arissian, et al., Phys. Rev. Lett. 105, 133002 (2010).

[22] E. S. Sarachik and G. T. Schappert, Phys. Rev. D 1, 2738-2753 (1970).

[23] H. R. Reiss and N. Hatzilambrou, J. Mod. Optics 53, 221-231 (2006).

[24] H. R. Reiss and V. P. Krainov, Phys. Rev. A 50, R910-R912 (1994); 74, 049903(E) (2006).

[25] H. R. Reiss, Phys. Rev. A 87, 033421 (2013).

[26] C. T. L. Smeenk, L. Arissian, B. Zhou, A. Mysyrowicz, D. M. Villeneuve, A. Staudte, and P. B. Corkum, Phys. Rev. Lett. 106, 193002 (2011).

[27] H. R. Reiss, Phys. Rev. A 76, 033404 (2007).

[28] B. Bergues, Y. Ni, H. Helm, and I. Yu. Kiyan, Phys. Rev. Lett. 95, 263002 (2005).

[29] H. R. Reiss, Phys. Rev. A 22, 1786-1813 (1980).

[30] L. V. Keldysh, Sov. Phys. JETP 20, 1307-1314 (1965). 